\title{
Genome-wide analysis of valine-glutamine motif-containing proteins related to abiotic stress response in cucumber (Cucumis sativus L.)
}

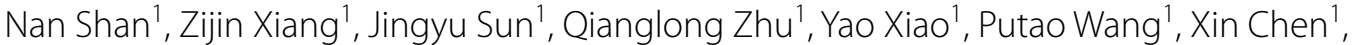 \\ Qinghong Zhou ${ }^{1 *}$ and Zengyu Gan ${ }^{1,2^{*}}$
}

\begin{abstract}
Background: Cucumber (Cucumis sativus L.) is one of the most important economic crops and is susceptible to various abiotic stresses. The valine-glutamine (VQ) motif-containing proteins are plant-specific proteins with a conserved "FxxhVQxhTG" amino acid sequence that regulates plant growth and development. However, little is known about the function of VQ proteins in cucumber.

Results: In this study, a total of 32 CsVQ proteins from cucumber were confirmed and characterized using comprehensive genome-wide analysis, and they all contain a conserved motif with 10 variations. Phylogenetic tree analysis revealed that these CsVQ proteins were classified into nine groups by comparing the CsVQ proteins with those of Arabidopsis thaliana, melon and rice. CsVQ genes were distributed on seven chromosomes. Most of these genes were predicted to be localized in the nucleus. In addition, cis-elements in response to different stresses and hormones were observed in the promoters of the CSVQ genes. A network of CSVQ proteins interacting with WRKY transcription factors (CsWRKYs) was proposed. Moreover, the transcripts of CsVQ gene were spatio-temporal specific and were induced by abiotic adversities. CsVQ4, CsVQ6, CsVQ16-2, CsVQ19, CsVQ24, CsVQ30, CsVQ32, CsVQ33, and CsVQ34 were expressed in the range of organs and tissues at higher levels and could respond to multiple hormones and different stresses, indicating that these genes were involved in the response to stimuli.
\end{abstract}

Conclusions: Together, our results reveal novel VQ resistance gene resources, and provide critical information on CSVQ genes and their encoded proteins, which supplies important genetic basis for VQ resistance breeding of cucumber plants.

Keywords: VQ motif, CsVQ proteins, Plant growth, Abiotic stress

\section{Background}

Cucumber (Cucumis sativus L.) is one of the most important economic crops worldwide and is popular and

\footnotetext{
*Correspondence: qinghongzhou@126.com; ganzy@jxau.edu.cn ${ }^{1}$ Agronomy College, Jiangxi Agricultural University, Nanchang 330045 China

2 Jiangxi Key Laboratory for Postharvest Technology and Nondestructive Testing of Fruits and Vegetables, Collaborative Innovation Center of Postharvest Key Technology and Quality Safety of Fruits and Vegetables, Jiangxi Agricultural University, Nanchang 330045, China
}

favored by consumers for its distinct aromas and flavors.

However, cucumber is vulnerable to various abiotic stresses, such as drought, low temperature, and salt during the growth and development process. Plants have gradually developed a complex set of mechanisms to adapt to adversity $[1,2]$, including the regulation of characteristic genes [3]. Valine-glutamine (VQ)-motif-containing proteins, characterized by a highly conserved core sequence FxxhVQxhTG (h denotes hydrophobic amino acid, $x$ means any amino acid), were reported to play crucial roles in plant growth, development, and responses 
to stresses [4]. The first VQ protein, AtVQ23 (formerly named SIB1, sigma factor binding protein1), was discovered from Arabidopsis [5]. Subsequently, members of $V Q$ gene family have been discovered and characterized in diverse plants, including Arabidopsis (34) [6], rice (40) [7], soybean (74) [8], Chinese cabbage (29) [9], bamboo (61) [10], tomato (26) [11], strawberry (25) [12], tea (25) [13], apple (49) [14], grapevine (18) [15], and melon (30) [16]. Based on the sequence features and homology of the VQ domains, VQ family members were classified into seven $[7,9,10,12,13]$ or ten $[11,14-18]$ subfamilies.

Accumulated evidences have demonstrated that VQ proteins serve as critical regulators in multiple biological processes, including development of endosperm and pollen [19-21], seed germination and early seedling establishment [22], photomorphogenesis [23], chloroplast development or photosystem assembly [6], and jasmonic acid (JA) or salicylic acid (SA) -mediated disease resistance [24-27]. In addition to these, lots of $V Q$ genes have been evidenced to play versatile functions in response to abiotic stresses [4]. Evidences show that AtVQ9 and AtVQ15 negatively regulate salt and osmotic tolerance in Arabidopsis [28, 29]. Similarly, $M a V Q 5$ gene in banana represses the transactivation of JA biosynthetic genes in response to cold stress [30]. SlVQ6-overexpressing plants exhibited a high sensitivity to high temperature stress, indicated that SlVQ6 gene in tomato negatively regulates thermotolerance [11]. In contrast, $P e V Q 28$ was found to act as a positive regulator for salt stress and abscisic acid (ABA) signal pathway [31]. IbVQ4 may participate in the drought and salt tolerance in sweet potato [32]. ZmVQ genes are responsive to abiotic stress [33], and PeVQ genes are differentially regulated by polyethylene glycol (PEG), ABA, and SA treatment in Moso bamboo [10].

Many VQ proteins participate in stress resistance by interacting with other proteins, especially with WRKY transcription factors [4]. As the largest family of transcriptional regulators in plants, WRKY transcription factors regulate plant biological processes and respond to various biotic and abiotic stresses by interacting with the conserved V and Q residues of the VQ proteins [34]. It was reported that AtVQ10 interacts with WRKY8 to modulate the basal defense against Botrytis cinerea in Arabidopsis [35]. Apple MdVQ protein was shown to bind with Group I and IIc MdWRKYs [14]. Another mode of VQ proteins is as a downstream substrate of mitogen-activated protein kinases (MAPKs). Previous studies showed that VQ proteins seem to bridge MAPK and WRKY transcription factors to form a ternary complex (WRKY-VQ-MAPK). AtVQ21/ MKS1 is first identified to be phosphorylated by AtMPK4, and AtVQ21, AtMPK4 and specific WRKY transcription factors (WRKY25 and WRKY33) co-regulate plant resistance through the complex interaction [18]. Tomato SIVQ6 has identified as a substrate of SIMPK1 and responds to abiotic stresses such as drought, high temperature and salt stresses [11]. In addition, other regulatory mechanisms of VQ proteins are increasingly being revealed. AtVQ29 restrains seedling de-etiolation by coacting with phytochrome-interacting factor1 [23]. During seed germination, AtVQ18 and AtVQ26 interact with the transcription factor $A B A-i n s e n s i t i v e 5$ to negatively regulate the ABA response in Arabidopsis [22]. The ring-type E3 ubiquitin ligase JUL1 (JAV1-associated ubiquitin ligase1) aims the VQ-motif protein to coordinate JA signaling pathway [36].

Although extensive efforts have been performed to investigate molecular mechanism of VQ protein involved in abiotic stress response in various plants, only a few of these genes have been characterized functionally to date and a large number of the VQ family members have not yet been studied. Particularly, $V Q$ genes in cucumber have been reported little. The draft genome sequence of cucumber, assembled using a combination of traditional Sanger and next-generation Illumina GA sequencing technologies, was obtained in 2009 [37], which affords insight into traits such as its stress resistance. However, some drawbacks, including the redundancy of repetitive DNA sequences, were found in the first assembly of the cucumber genome. Based on the RNA-Seq reads, the prediction of protein-coding genes was improved in the reassembled cucumber genome (cucumber 'Chinese Long' v2 genome) [38]. In recent years, with the development of new cost-effective and accurate technologies [39], including 10X Genomics, optical mapping, and high-throughput chromosome conformation capture, a chromosome-scale genome assembly of cucumber (cucumber 'Chinese Long' v3 genome) was generated [40], which serves as a valuable resource for genetic research in cucumber.

The completion and improvement of cucumber genome sequence provides an excellent opportunity for genome-wide analysis of $V Q$ gene family. In the current study, a total of $32 \mathrm{Cs} V Q$ genes were identified based on cucumber genome (Cucumber 'Chinese Long' v2) and transcriptome databases of cucumber. These $C s V Q$ genes were clustered into 9 subfamilies based on phylogenetic analysis. Then their conserved motifs, conserved domains, gene structure, functional interaction network, and spatio-temporal expression patterns were comprehensively studied. To identify $C s V Q$ candidate genes associated with abiotic stress, transcripts of nine $C s V Q$ genes were examined under cold, drought, salinity and hormone treatments. These results indicated that CsVQ proteins not only involved in plant growth and development regulation, but also in abiotic stress and hormone 
treatment. In summary, this study would provide comprehensive information about CsVQ genes from cucumber, as well as the insights for the further functional investigation and application of novel CsVQ candidate genes for crop improvement, especially in aspects of stress resistance, growth and development.

\section{Results}

Gene characterization, phylogenetic tree, and chromosomal locations analysis

To identify the homologous VQ protein family in cucumber plants, conserved motif (PF05678) of VQ protein was used as a query in Cucumber 'Chinese Long' v2 genome of Cucurbit Genomics Database (CuGenDB) [37]. A total of $32 C s V Q$ genes were identified and assigned specific names based on sequence similarity and phylogenetic tree among AtVQ [6], CmVQ [16], SIVQ [11], OsVQ [7], and CsVQ proteins (Fig. 1; Table 1). Protein sequence analysis results showed that all CsVQ proteins shared similar amino acid sequence FxxxVQxL/F/VTG, and four types were identified in cucumber, including Fxx $\mathrm{VQx}$ VTG (1/32), FxxxVQxLTA (1/32), FxxxVQxFTG (6/32), and FxxxVQxLTG (24/32) (Fig. 1). Gene locus ID, open reading frame length, and physiological and biochemical properties of $32 \mathrm{CsVQ}$ proteins were analyzed, including length ranging from 81 to 405 amino acids (aa), molecular weight ranging from 9.28 to $42.78 \mathrm{kDa}$, and theoretical isoelectric point (pI) ranging from 4.37 to 11.80 (Table 1). Furthermore, subcellular localization prediction results showed that most CsVQ proteins were located in the nucleus, whereas a few proteins were located in the mitochondria or cytoplasm (Table 1).

To explore the evolutionary relationship between cucumber CsVQ proteins, a phylogenetic tree was constructed among 32, 30, 34, 40, and 26 VQ proteins of cucumber, melon, Arabidopsis, rice, and tomato, respectively (Fig. 2A). CsVQ proteins of cucumber appeared in 9 groups (I, II, IV-X) according to the classification of Arabidopsis, melon and tomato in the previous studies $[11,16,18]$. Group IX possessed largest number of VQ proteins, including 28 VQ proteins (6 CsVQs, $6 \mathrm{CmVQs}$, 6 AtVQs, 6 OsVQs, and 4 SlVQs). Group V, the second largest group, consist of $25 \mathrm{VQ}$ proteins (6 CsVQs, 5 CmVQs, 5 AtVQs, 5 OsVQs, and 4 tomato SIVQs). There was only one CsVQ protein in group VII (Fig. 2A). The evolutionary relationship indicates that the CsVQ proteins exhibit a close relationship with the melon and Arabidopsis VQ proteins and a distant relationship with the rice VQ proteins in the same group.

Chromosomal location results showed that $32 \mathrm{CsVQ}$ genes were randomly and unequally distributed on 7 chromosomes. Specifically, chromosomes 3 and 4 harbored the largest number of $C s V Q$ genes with eight genes, and chromosomes 2 and 6 both possessed six CsVQ genes. However, chromosomes 5 and 7 had only one gene each (Fig. 2B). Gene structure analysis results showed that most CsVQ genes possessed one exon structure, except that CsVQ2, CsVQ4, CsVQ7, CsVQ9, $C s V Q 25$, and $C s V Q 27$ contained two exon structures (Fig. S1).

\section{Conserved motif analysis of CsVQ proteins}

VQ- motif was reported to have a significant impact on the function of VQ proteins, and the site mutation of VQ motif may affect the functions [4]. To investigate the sequence features of CsVQ proteins, conserved motifs were predicted and analyzed using the MEME suit. Ten motifs, ranging from 10 to 38 amino acids, were predicted, and exhibited highly conserved amino acid residues (Fig. 3; Fig. S1). Motif 1 corresponding to the VQ-containing motif was distributed in all CsVQ proteins, and other conserved motifs (motifs 2 to 10) were unequally distributed in CsVQ proteins; e.g., motif 2 in six, motif 3 in ten, motif 4 in four, motif 5 in five, motif 6 in five, motif 7 in two, motif 8 in five, motif 9 in six, and motif 10 in three CsVQ proteins (Fig. 3). Some motifs were present in the proteins of specific groups. For example, motifs 2 and 10 were specifically distributed in CsVQ4, CsVQ13, and CsVQ19 belonging to Group I; motif 4 was specifically distributed in proteins belonging to Group II; motifs 7 and 9 were specifically distributed in CsVQ2, CsVQ3, CsVQ8, CsVQ20, CsVQ21-1, and CsVQ21-2 belonging to Group V; and motif 5 was specifically distributed in proteins belonging to Group IX (Fig. 3). In addition, CsVQ proteins containing similar motifs belonged to the same group, which corresponded with the phylogenetic analysis results (Fig. 2A). Conversely, CsVQ proteins in different groups exhibited significantly different types and numbers of motifs, indicating the structural basis for diversity in protein function.

\section{Interaction network of CsVQ proteins}

Interacting with the WRKY family is the common mode of action of VQ proteins. An interaction network of CsVQ proteins was constructed to understand their functional and physical interactions. Fourteen CsVQ proteins and twelve CsWRKY proteins were shown to participate in the interaction network (Fig. 4). To further explore the network, a specific CsVQ-CsWRKY interaction network was constructed (Fig. 5). Nine key nodes, including CsVQ2, CsVQ3, CsVQ6, CsVQ9, CsVQ14, CsVQ19, CsVQ211, CsVQ21-2, and CsVQ32, are presumed to interact with different WRKY transcription factors (Fig. 5A). For instance, CsVQ6 was predicted to interact with 
A

Conserved Motif

FxxxVQxxTg

\begin{tabular}{|c|c|}
\hline $\begin{array}{l}\text { CsVQ1 } \\
\text { CsVQ2 } \\
\text { CsVQ3 } \\
\text { CsVQ4 } \\
\text { CsVQ14 } \\
\text { CsVQ6 } \\
\text { CsVQ7 } \\
\text { CsVQ8 } \\
\text { CsVQ9 } \\
\text { CsVQ10 } \\
\text { CsVQ11 } \\
\text { CsVQ12 } \\
\text { CsVQ13 } \\
\text { CsVQ32 } \\
\text { CsVQ33 } \\
\text { CsVQ16-2 } \\
\text { CsVQ25 } \\
\text { CsVQ21-2 } \\
\text { CsVQ19 } \\
\text { CsVQ20 } \\
\text { CsVQ21-1 } \\
\text { CsVQ22 } \\
\text { CsVQ23-2 } \\
\text { CsVQ24 } \\
\text { CsVQ16-1 } \\
\text { CsVQ23-1 } \\
\text { CsVQ27 } \\
\text { CsVQ28 } \\
\text { CsVQ29 } \\
\text { CsVQ34 } \\
\text { CsVQ31 } \\
\text { CsVQ30 }\end{array}$ & 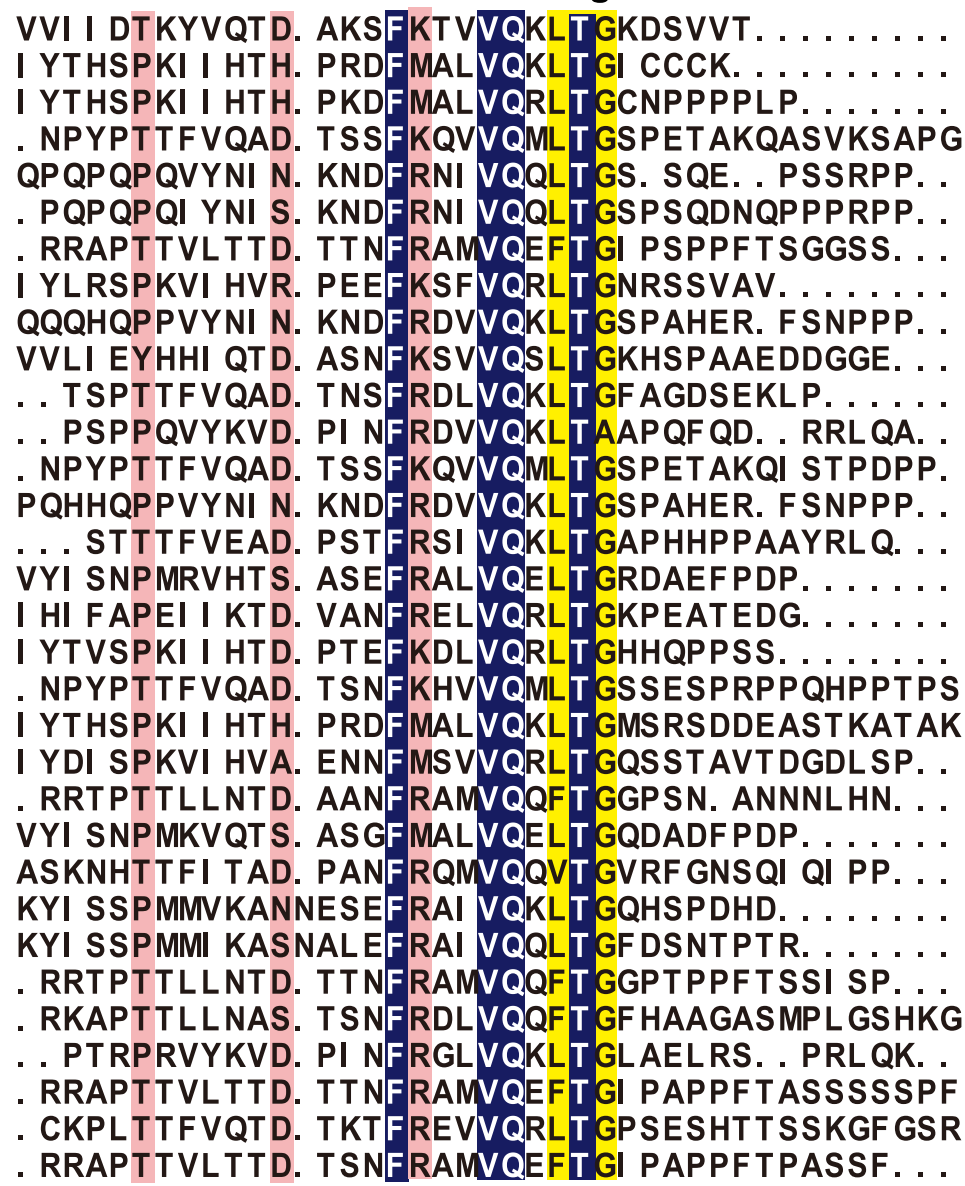 \\
\hline
\end{tabular}

B

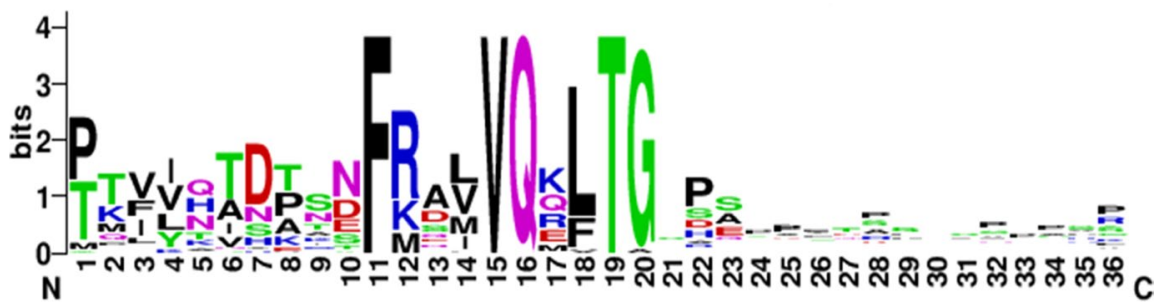

Fig. 1 Multiple sequence alignment of CsVQ proteins in cucumber. A, sequence alignment of CsVQ proteins using DNAMAN version 6.0, where $\mathrm{x}$ represents any amino acid. The conserved amino acid sequence of FxxXVQxLTG is clearly highly conserved and shown in (B)

CsWRKY16, CsWRKY16, and CsWRKY17; CsVQ14 with CsWRKY16 and CsWRKY24; CsVQ21-1 and CsVQ21-2 with CsWRKY2, CsWRKY15, CsWRKY16, CsWRKY17, CsWRKY23, and CsWRKY39. In addition, 12 CsWRKYs were speculated to interact with different CsVQ proteins, including CsWRKY15, CsWRKY16, CsWRKY28, CsWRKY39, CsWRKY43, CsWRKY46, CsWRKY53, and CsWRKY66 belong to group IIc
WRKY transcription factor and the others belong to the group I WRKY transcription factor [41] (Fig. 5A). In Arabidopsis, AtWRKY20, AtWRKY25, AtWRKY33, and AtWRKY51 were reported to closely interact with different AtVQ proteins [11]. Multiple sequence alignment of C-terminal WRKY domains of above CsWRKY and AtWRKY proteins showed that the core binding domain of these WRKYs was highly conserved (Fig. 5B), 
Table 1 Properties of the cucumber VQ genes and proteins

\begin{tabular}{|c|c|c|c|c|c|c|c|c|}
\hline \multirow[t]{2}{*}{ Gene Name } & \multirow[t]{2}{*}{ Gene Locus } & \multirow[t]{2}{*}{ ORF length (bp) } & \multirow[t]{2}{*}{ Chr. No. } & \multirow[t]{2}{*}{ Position } & \multicolumn{4}{|l|}{ Protein } \\
\hline & & & & & Length (aa) & MW (kDa) & $\mathrm{pl}$ & Subcellular location \\
\hline CsVQ1 & Csa4G075740 & 246 & 4 & $5,375,816 . .5,376,158(+)$ & 81 & 9.28 & 9.72 & Nucleus \\
\hline CsVQ2 & Csa1G023620 & 645 & 1 & $2,381,813 . .2,382,555(+)$ & 214 & 22.99 & 6.49 & Nucleus \\
\hline CsVQ3 & Csa5G609750 & 432 & 5 & $23,658,353 . .23,658,886(+)$ & 143 & 15.82 & 8.76 & Nucleus \\
\hline CsVQ4 & Csa1G074920 & 759 & 1 & $7,652,045 . .7,655,640(+)$ & 252 & 27.41 & 10.37 & Nucleus \\
\hline CsvQ6 & Csa3G011640 & 969 & 3 & $1,171,817 . .1,173,439(+)$ & 322 & 34.98 & 11.80 & Cytoplasm \\
\hline CsVQ7 & Csa3G895870 & 768 & 3 & $38,675,299$.. 38,676,094 (-) & 255 & 27.48 & 8.49 & Nucleus \\
\hline CsVQ8 & Csa3G785410 & 339 & 3 & $30,519,688 . .30,520,134(+)$ & 112 & 12.33 & 11.53 & Extracell; Mitochondrion \\
\hline CsVQ9 & Csa2G369080 & 1074 & 2 & $17,972,789 . .17,973,921(-)$ & 357 & 38.62 & 10.89 & Nucleus \\
\hline CsVQ10 & Csa6G523420 & 375 & 6 & $28,092,380 . .28,092,798(-)$ & 124 & 14.08 & 7.73 & Nucleus \\
\hline CsVQ11 & Csa4G651800 & 528 & 4 & $22,459,448$.. 22,460,042(+) & 175 & 19.27 & 10.96 & Nucleus \\
\hline CsVQ12 & Csa2G271490 & 267 & 2 & $13,149,552 . .13,149,818(+)$ & 88 & 9.74 & 10.45 & Nucleus \\
\hline CsVQ13 & Csa6G517410 & 675 & 6 & $27,253,151 . .27,253,923(+)$ & 224 & 24.34 & 8.94 & Nucleus \\
\hline CsVQ14 & Csa3G902300 & 981 & 3 & $38,997,555$.. 38,998,831 (+) & 326 & 35.22 & 10.52 & Extracell \\
\hline CsVQ16-1 & Csa2G010160 & 423 & 2 & $1,842,928$.. 1,843,350 (-) & 140 & 15.73 & 5.90 & $\begin{array}{l}\text { Cytoplasm; Extracell; } \\
\text { Mitochondrion; Nucleus }\end{array}$ \\
\hline CsVQ16-2 & Csa3G820480 & 435 & 3 & $31,922,073 . .31,922,792(-)$ & 144 & 16.41 & 4.37 & Nucleus \\
\hline CsVQ19 & Csa4G637130 & 741 & 4 & $20,716,210 . .20,717,384(-)$ & 246 & 36.42 & 9.08 & Nucleus \\
\hline CsVQ20 & Csa2G350280 & 714 & 2 & $15,927,811 \ldots 15,928,547(+)$ & 237 & 25.10 & 6.50 & Nucleus \\
\hline CsVQ21-1 & Csa2G302100 & 639 & 2 & $14,531,424 \ldots 14,532,251(-)$ & 212 & 22.96 & 7.84 & Nucleus \\
\hline CsVQ21-2 & Csa6G338080 & 534 & 6 & $15,427,318 . .15,427,851(-)$ & 177 & 19.37 & 6.90 & Nucleus \\
\hline CsVQ22 & Csa4G618460 & 582 & 4 & $19,753,678 . .19,754,725(+)$ & 193 & 20.77 & 9.13 & Nucleus \\
\hline CsVQ23-1 & Csa2G010150 & 420 & 2 & $1,836,083 . .1,836,502(-)$ & 139 & 16.05 & 8.35 & Nucleus \\
\hline CsVQ23-2 & Csa4G431960 & 495 & 4 & $16,320,651$.. 16,321,291(+) & 164 & 17.52 & 4.39 & Nucleus \\
\hline CsVQ24 & Csa3G816710 & 723 & 3 & $31,696,876 . .31,697,690(-)$ & 240 & 25.98 & 6.51 & $\begin{array}{l}\text { Cytoplasm; Extracell; } \\
\text { Nucleus }\end{array}$ \\
\hline CsVQ25 & Csa3G807350 & 1035 & 3 & $30,912,350 . .30,913,425(-)$ & 344 & 34.35 & 4.96 & Cytoplasm; Nucleus \\
\hline CsVQ27 & Csa4G056730 & 690 & 4 & $4,862,111 . .4,862,837(-)$ & 229 & 24.60 & 6.35 & Extracell; Nucleus \\
\hline CsVQ28 & Csa6G526440 & 564 & 6 & $28,778,536 . .28,779,301(-)$ & 187 & 20.15 & 6.97 & Nucleus \\
\hline CsVQ29 & Csa6G495810 & 507 & 6 & $24,113,317$.. 24,113,823(+) & 168 & 18.53 & 9.57 & Nucleus \\
\hline CsVQ30 & Csa6G500500 & 1197 & 6 & $24,943,199 . .24,944,684(-)$ & 398 & 42.56 & 7.28 & Nucleus \\
\hline CsVQ31 & Csa4G448640 & 501 & 4 & $16,849,697 . .16,850,197(+)$ & 166 & 18.80 & 10.08 & Nucleus \\
\hline CsVQ32 & Csa3G889820 & 1011 & 3 & $37,789,436 . .37,791,027(-)$ & 336 & 36.12 & 10.89 & Nucleus \\
\hline CsVQ33 & Csa7G432350 & 423 & 7 & $17,187,689 . .17,188,487(+)$ & 140 & 15.09 & 7.04 & Nucleus \\
\hline CsVQ34 & Csa4G028990 & 1218 & 4 & $3,005,474 . .3,007,138(+)$ & 405 & 42.78 & 6.85 & Nucleus \\
\hline
\end{tabular}

which were considered to be the key to the interaction between VQ and WRKY proteins.

\section{Expression profiles of CsVQ genes in different tissues of cucumber plant}

To understand the potential function of CsVQ genes, cis-elements were predicted. Four types of promoters, including stress-responsive, hormone-responsive, tissuespecific expression, as well as light-responsive promoters, were identified in CsVQ genes (Table S1). Stress-responsive elements responded to drought, low temperature, defense and stress, and anaerobic induction (Table S1).
Elements were also found to respond to hormones, such as ABA, SA, methyl jasmonate (MeJA), auxin, and gibberellin, indicating that $C s V Q$ genes may be regulated by these hormones (Table S1; Fig. S2). Additionally, the promoters of some CsVQ genes comprised W-box elements (TTGAC, WRKY-binding sites), suggesting that their transcriptions may be regulated by WRKY proteins (Table S1).

To clarify the specificity of tissue expression, the expression levels of each $C s V Q$ gene from $\mathrm{CuGenDB}$ were examined. The $C s V Q$ genes were differentially 


\section{A}

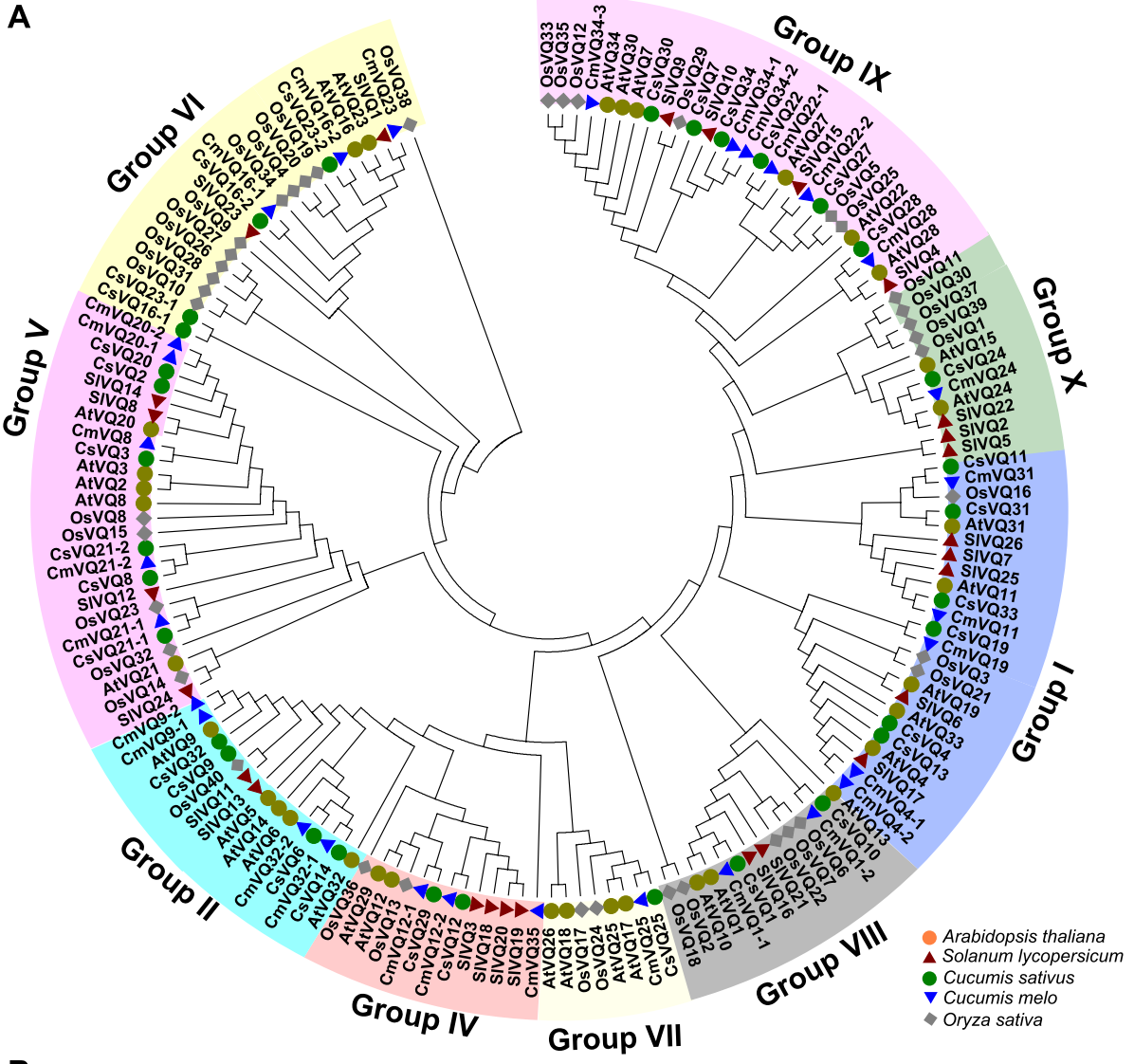

B

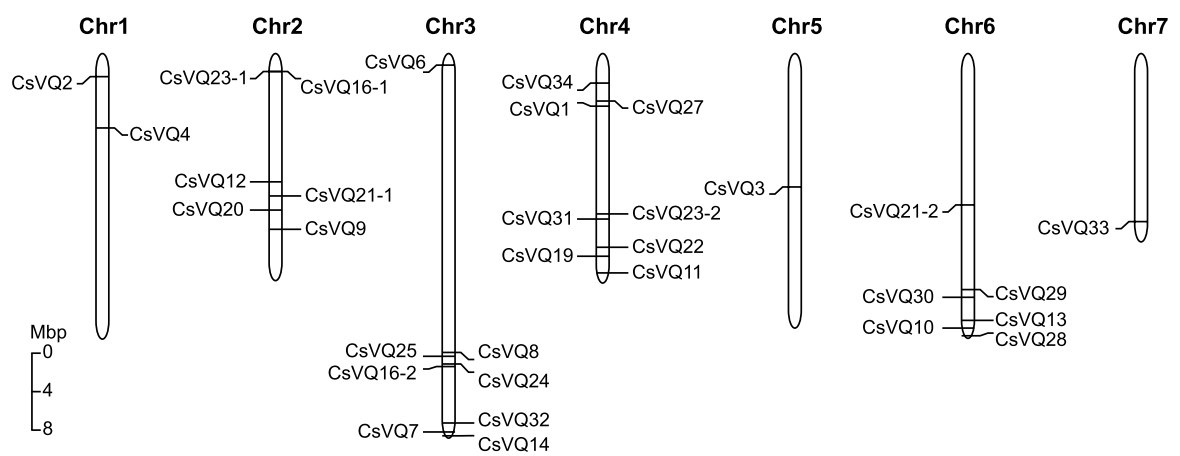

Fig. 2 Phylogenetic tree (A) and chromosomal locations (B) of CsVQ genes. A, the phylogenetic tree of the 32 cucumber CsVQs, 30 melon CmVQs, 34 Arabidopsis AtVQs, 26 tomato SIVQs, and 40 rice OsVQs was established by using the maximum likelihood method in MEGA X. Proteins from cucumber, melon, Arabidopsis, tomato, and rice are denoted by green dots, blue triangles, brown dots, red triangles, and grey squares, respectively. B, the chromosomal locations of the CSVQ genes were determined using MapChart 2.3.2

expressed in the tissues of cucumber plants (Fig. 6). Among 32 CsVQ genes, CsVQ4, CsVQ6, CsVQ16-2, CsVQ19, CsVQ24, CsVQ30, CsVQ32, CsVQ33, and CsVQ34 were highly expressed, whereas CsVQ31 and $C s V Q 2$ had the lowest expression levels (Fig. 6). CsVQ24 and CsVQ16-2 exhibited the highest transcription levels in all $C s V Q$ genes from selected samples, which was also similar to the CsVQ gene expression levels in different tissues of the Chinese long cucumber species (data from bioproject PRJNA80169 of Cucurbit Expression Atlas).

Expression profiles of CsVQ genes in response to hormones To better understand the potential function of $C s V Q$ genes in plant hormones, the responses of CsVQ4, 


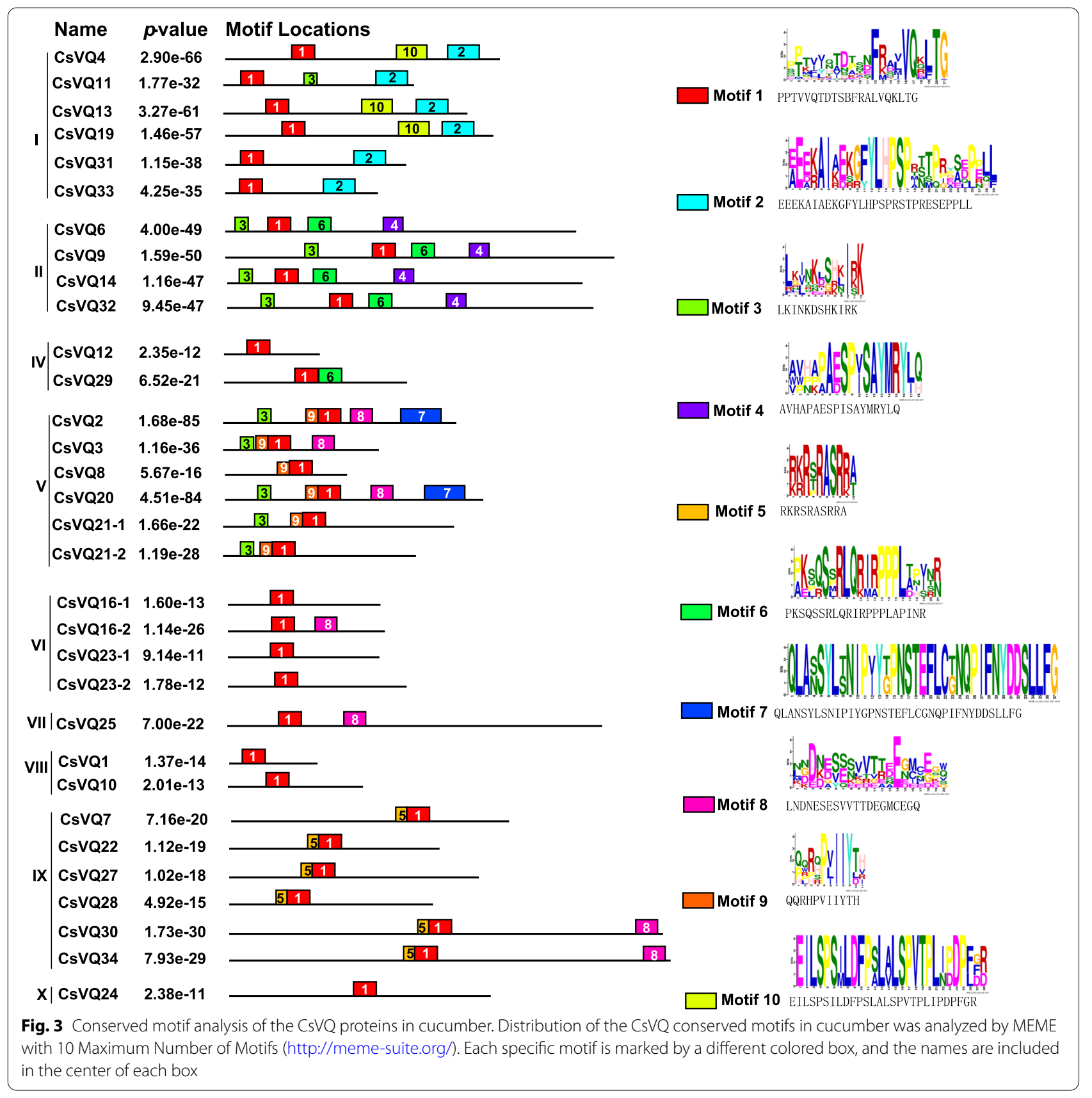

CsVQ6, CsVQ16-2， CsVQ19， CsVQ24， CsVQ30, CsVQ32, CsVQ33, and CsVQ34 to SA, ABA, and MeJA treatments were investigated using quantitative realtime polymerase chain reaction (qRT-PCR) (Fig. 7), based on their high expression in roots, stems, male flowers, female flowers, ovaries, and fruits (Fig. 6). The results showed that four $C s V Q$ genes, namely $C s V Q 4$, CsVQ6, CsVQ16-2, and CsVQ19, were induced by ABA treatment (Fig. 7A-D). Following ABA treatment, CsVQ4, CsVQ6, CsVQ16-2, and CsVQ19 were significantly upregulated at $3 \mathrm{~h}$ post-treatment, reaching a peak at $6 \mathrm{~h}$ after treatment, and three of the genes (CsVQ4, CsVQ6, and CsVQ19) maintained an evident downregulation tendency $9 \mathrm{~h}$ after $\mathrm{ABA}$ treatment (Fig. 7A-D). CsVQ16-2 expression was strongly promoted by ABA treatment, whereas CsVQ24, CsVQ30, CsVQ32, CsVQ33, and CsVQ34 were barely responsive to ABA treatment (Fig. 7E-I). During SA treatment, CsVQ16-2 and CsVQ24 were upregulated at all stages, and CsVQ4, CsVQ6, and CsVQ34 (except at 


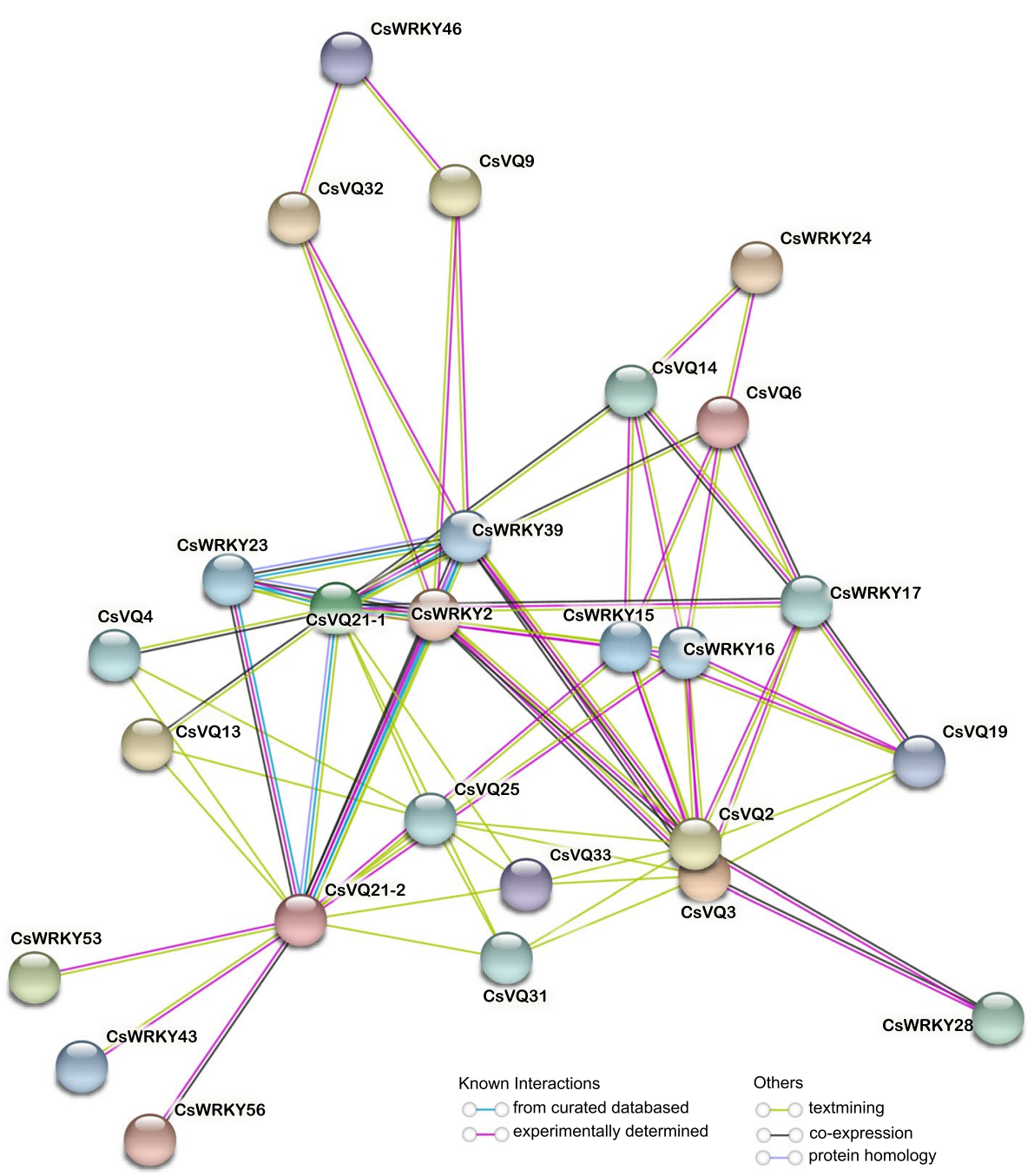

Fig. 4 Putative interaction network of CsVQ proteins in cucumber. The prediction of interaction between CsVQ proteins and CsWRKY proteins were integrated using the STRING tool (https://string-db.org/)

$3 \mathrm{~h}$ ) were significantly upregulated at $3 \mathrm{~h}, 9 \mathrm{~h}$, and $24 \mathrm{~h}$, whereas $C s V Q 19$ was slightly downregulated at $6 \mathrm{~h}$ and $12 \mathrm{~h}$ (Fig. 7). Nevertheless, compared to the beforetreatment levels, these nine CsVQ genes were highly expressed at $24 \mathrm{~h}$ after SA treatment (Fig. 7). Additionally, the expression levels of CsVQ4, CsVQ6, CsVQ162 , and CsVQ19 sharply decreased at $3 \mathrm{~h}$ and $6 \mathrm{~h}$ and significantly increased at $9 \mathrm{~h}, 12 \mathrm{~h}$, and $24 \mathrm{~h}$ after MeJA treatment, and CsVQ24, CsVQ30, and CsVQ34 were upregulated at all stages of the MeJA treatment (Fig. 7). CsVQ4 and CsVQ6 were upregulated 5-fold and 4-fold at $9 \mathrm{~h}$ post MeJA treatment, respectively, and CsVQ162 and $C s V Q 19$ were upregulated 10-fold and 2-fold at 24h after MeJA treatment (Fig. 7). These results indicate that almost all highly expressed $C s V Q$ genes are involved in the cucumber response to hormones; however, it is not known whether they are involved in the adversity response in cucumbers.

\section{Expression profiles of CsVQ genes in response to abiotic treatment}

To characterize the biological functions of the CsVQ genes under salt, drought, and low-temperature stress conditions, the expression levels of CsVQ4, CsVQ6, CsVQ16-2, CsVQ19, CsVQ24, CsVQ30, CsVQ32, CsVQ33, and CsVQ34 were detected (Fig. 8). When subjected to salt stress, the expression of CsVQ4, CsVQ6, CsVQ16-2, CsVQ19, and CsVQ24 was significantly upregulated compared to that of the control, although CsVQ4 transcripts decreased at $3 \mathrm{~h}$, and Cs VQ24 transcripts decreased at 9h (Fig. 8A-E). Compared with the control, all selected CsVQ genes were highly expressed at $24 \mathrm{~h}$ after salt stress (Fig. 8). The 


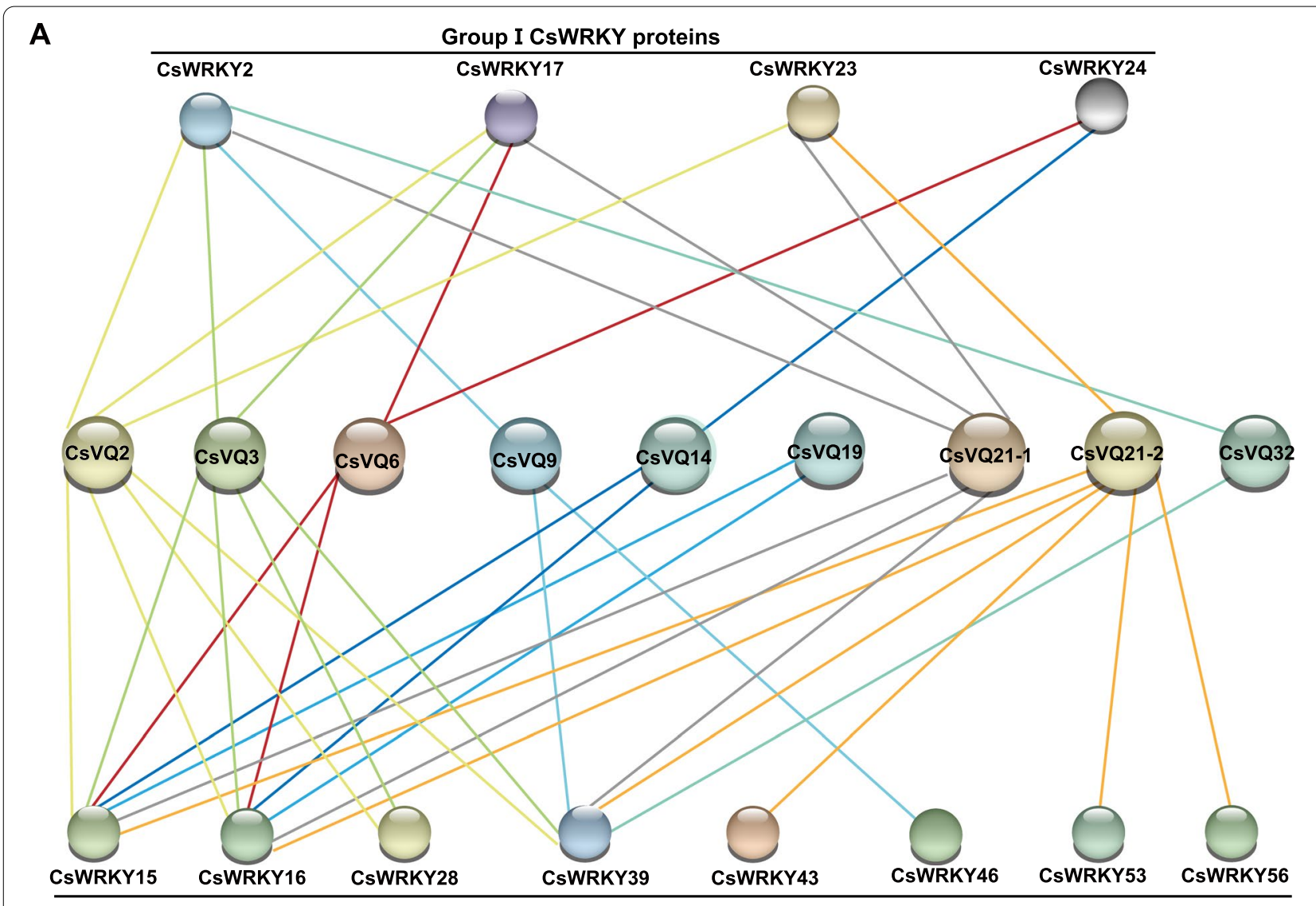

B

Group IIc CsWRKY proteins

AAWRKY20 REPRVVVQTLSEVDI LDDGYRWRKYGQKVVRGNPNPRSYYKCTAHGCPVRKHVERASHDPKAVI TTYEGKHDHD AtWRKY25 KEPRVVVQTI SDI DVLI DGFRWRKYGQKVVKGNT NPRSYYKCTFQGCGVKKQVERSAADERAVLTTYEGRHNHD - AtWRKY33 REPRI VVQTTSDI DI LDDGYRWRKYGQKVVKGNPNPRSYYKCTTI GCPVRKHVERASHDMRAVI TTYEGKHNHD 흔 CsWRKY2 REPRVVVQTTSDI DI LDDGYRWRKYGQKVVKGNPNPRSYYKCTNPGCPVRKHVERASHDLRAVI TTYEGKHNHD 屯ै CsWRKY17 REPRVVVQTLSEVDI LDDGYRWRKYGQKVVRGNPNPRSYYKCTNVGCPVRKHVERASHDPKAVI TTYEGKHNHD CSWRKY23 KEPRI VVQTTSEI DI LPDGYRWRKYGQKVVKGNPNPRSYYKCTSLGCPVRKHI ERAANDMRAVI TTYEGKHNHE CSWRKY24 NEHHAMAQDSTGI EI SGKGVRWRKYGQKVVKGNLYPRSYYRCTGLKCKARKYVERASEDPDSFI TTYEGKHNHG Consensus e q g r wrkgqkvign prsy ct c k er d t yegh h

AtWRKY51 RVAFRTRS. . KI DVMDDGF KWRKYGKKSVKNNI NKRNYYKCSSEGCSVKKRVERDGDDAAYVI TTYEGVHNHE ○ CSWRKY15 RVVVQTTS. . EVDI LDDGYRWRKYGQKVVKGNPNPRSYYKCTNPGCTVRKHVERASHDLKSVI TTYEGKHNHD CSWRKY16 RGSGDSMAGGACGTPSEDGYNWRKYGQKQVKGSEYPRSYYKCTHPNCQVKKKVER. SHEGHI TEI I YKGTHNHP CsWRKY28 RI AFQTKS. . VEDVLDDGYRWRKYGQKAVKHSNHPRSYYRCTHHTCNVKKQI QRHSKDPTI VVTTYEGI HNHP む) CsWRKY39 RI VVQTI S. . NVDKLDDGYWWRKYGQKVVKGNPNPRSYYKCTYPGCGVRKHI ERASHDFRAVVTTYEGKHNHD CSWRKY43 RFSFKTMT. . . DVDVLDDGYKWRKYGQKVVKNTLHPRSYYRCTEENCKVKKRVERLADDPRMVI TTYEGRHAHS CsWRKY46 RVAFMTKS. . EVDHLEDGYRWRKYGQKAVKNSAYPRSYYRCTTQKCGVKKRVERSYEDPSI VI TTYEGQHNHL CsWRKY53 RFSFKTMS. . . DVDVLDDGYKWRKYGQKVVKNT QHPRSYYRCTQDHCRVKKRVERLAEDPRMVI TTYEGRHVHS CSWRKY56 RYAFQTRS. . QVDI LDDGYRWRKYGQKAVKNNKF PRSYYRCTHQGCNVKKQVQRLTRDEGVVVTTYEGMHTHS dg wr kyg k vk $\mathbf{r}$ y y c c v k r y $\mathrm{g} \mathrm{h} h$

Fig. $\mathbf{5}$ Interaction of CsVQ proteins with CsWRKY transcription factor in cucumber. A, the prediction of interaction between CsVQ proteins and CsWRKY proteins was drawn by the PAIR website. B, sequence analysis of the C-terminal WRKY domains of group I and group IIc CsWRKY proteins [41]

expression of CsVQ4, CsVQ6, CsVQ16-2, CsVQ19, and $C s V Q 24$ were upregulated after exposure to drought and cold stresses, although the expression of certain genes started declining at 12 and $24 \mathrm{~h}$ (Fig. 8AE). The expression levels of CsVQ16-2 significantly increased under the three stresses, which were upregulated by 35 times at $24 \mathrm{~h}$ after salt treatment, 30 


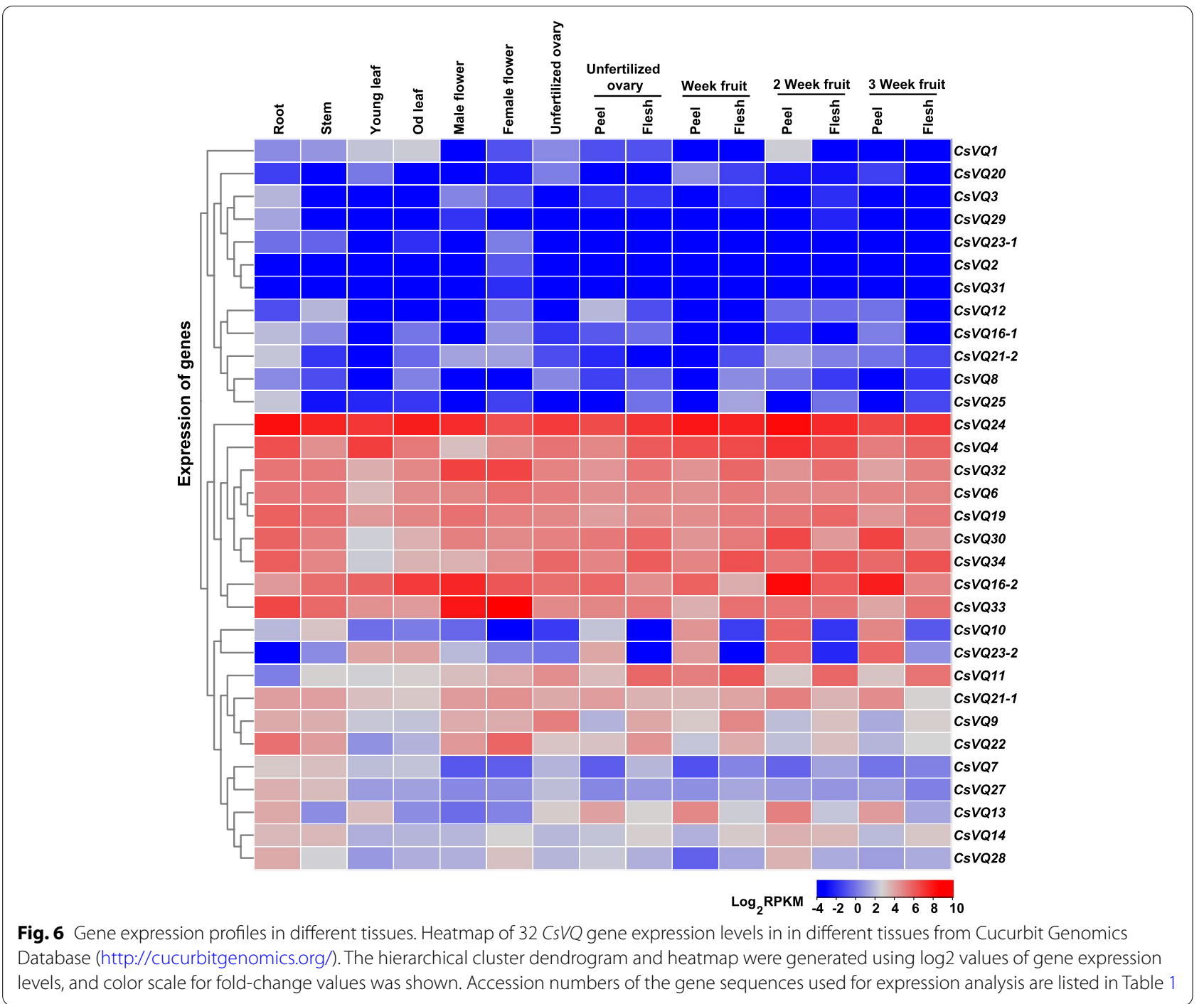

times at $24 \mathrm{~h}$ after drought treatment, and 25 times at $12 \mathrm{~h}$ after cold treatment, indicating that $C s 16-2$ is involved in the response to different environmental stresses, such as salt, drought, and low temperature and may play diverse roles in the adversity response in cucumbers.

Among the three abiotic treatments, the response of CsVQ4 to cold stress was the most noticeable relative to that to drought and salt stresses, with a 15 -fold increase at $12 \mathrm{~h}$ after cold treatment (Fig. 8). Moreover, CsVQ6 and CsVQ19 were more sensitive to salt and cold stresses than to the drought stress, whereas CsVQ16-2 responded drastically to all three stresses (Fig. 8). These results suggest that almost all selected
CsVQ genes are involved in the salt and drought responses of cucumber plants, and the response mechanisms are complex and diverse.

\section{Discussion}

Cucumber is one of the most widely cultivated vegetables in the world, its growth and development were significantly affected by genetic and environmental factors [42]. VQ protein is a plant-specific transcription factor and is involved in plant growth, development, and responses to environmental adversities. In previous studies, the $V Q$ gene family has been identified and characterized in Arabidopsis [6], tomato [11], rice [7], soybean [8], Chinese cabbage [9], bamboo [10], strawberry [12], 


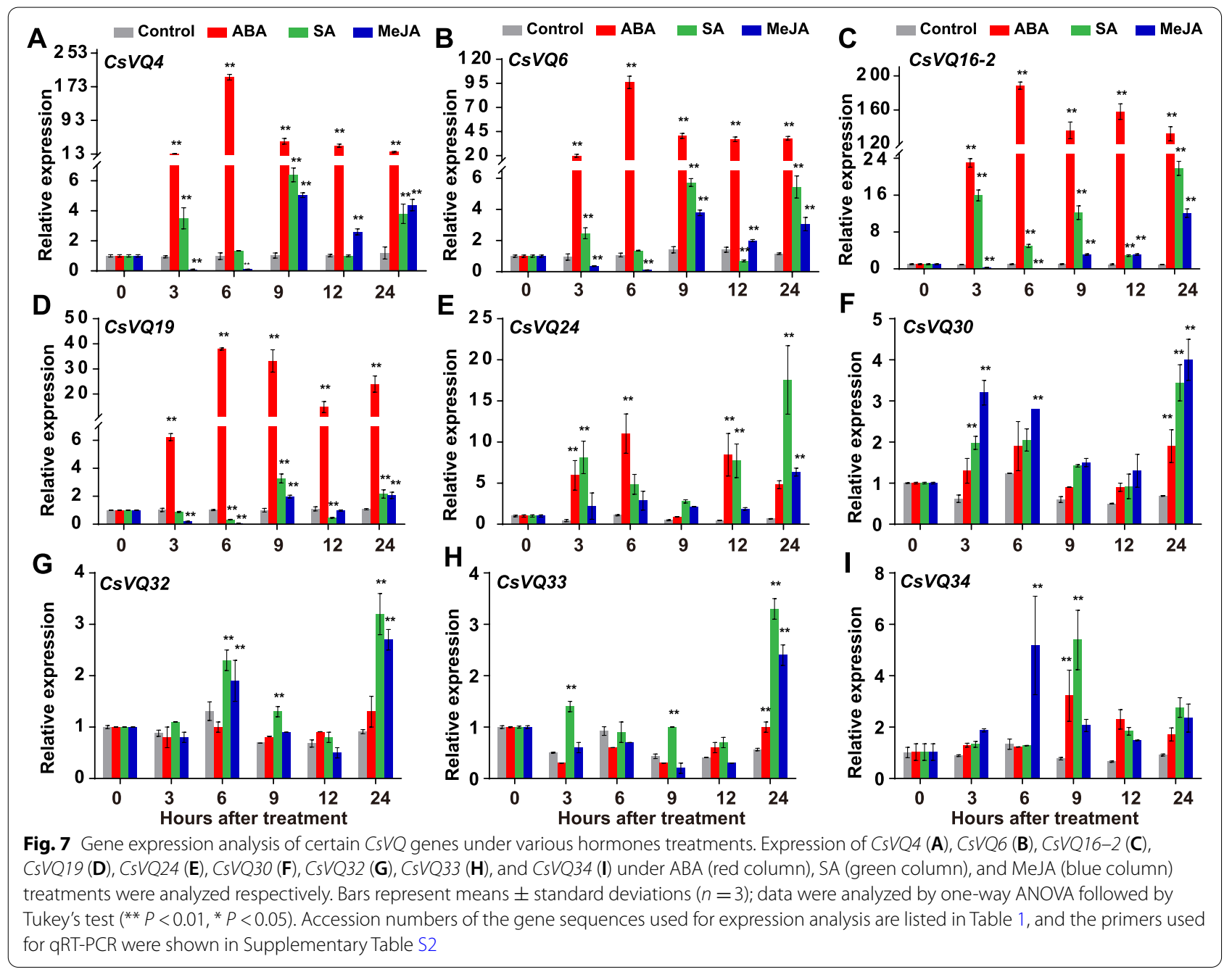

tea [13], apple [14], grapevine [15] and melon [16]. However, detailed information concerning CsVQ characters and functions, particularly their role in stresses responses of cucumber, remained unclear. In the present study, 32 $C s V Q$ genes were identified in $\mathrm{CuGenDB}$, and the structure and characteristics of $C s V Q$ genes and proteins were analyzed. The VQ protein is characterized by the highly conserved amino acid sequence FxxxVQxL/F/VTG, with differences in the regions of leucine (L) and glycine (G). In previous studies, six motifs were identified in Arabidopsis [6], five in soybean [43], six in Chinese cabbage [9], six in maize [33], five in Moso bamboo [10], five in strawberry [44], two in tea plant [13], four in melon [16], and seven in tomato [11]. Sequence analysis of CsVQ protein showed that all $C s V Q$ proteins shared a similar VQ domain with four variations (LTG, FTG, VTG, LTA) (Fig. 1), which was the same as that of melon [16]. Furthermore, the VQ domain can affect protein-protein interactions [45], and the mutation of the VQ amino acid residue may invalidate the interaction between VQ and transcription factors [20, 25, 34].

The Arabidopsis VQ protein has been classified into 10 groups based on the evolutionary analysis results [6]. Based on the phylogenetic tree analysis, CsVQ proteins can be divided into nine groups, and there were no members in group III, as in melon [16]. The conserved motifs provided further support for the classification of $C s V Q$ genes in cucumber (Fig. 3). The most closely related CsVQ members in the same group showed similarities in motif distribution (Fig. 3). Ten conserved motifs were discovered in all CsVQ proteins, and the conserved motifs were significantly different among different groups of CsVQ proteins. Most CsVQ proteins of the same group apparently had similar motifs constituents (Fig. 3), suggesting that the similarities in conserved motifs in the same subfamily corroborate their classification and 

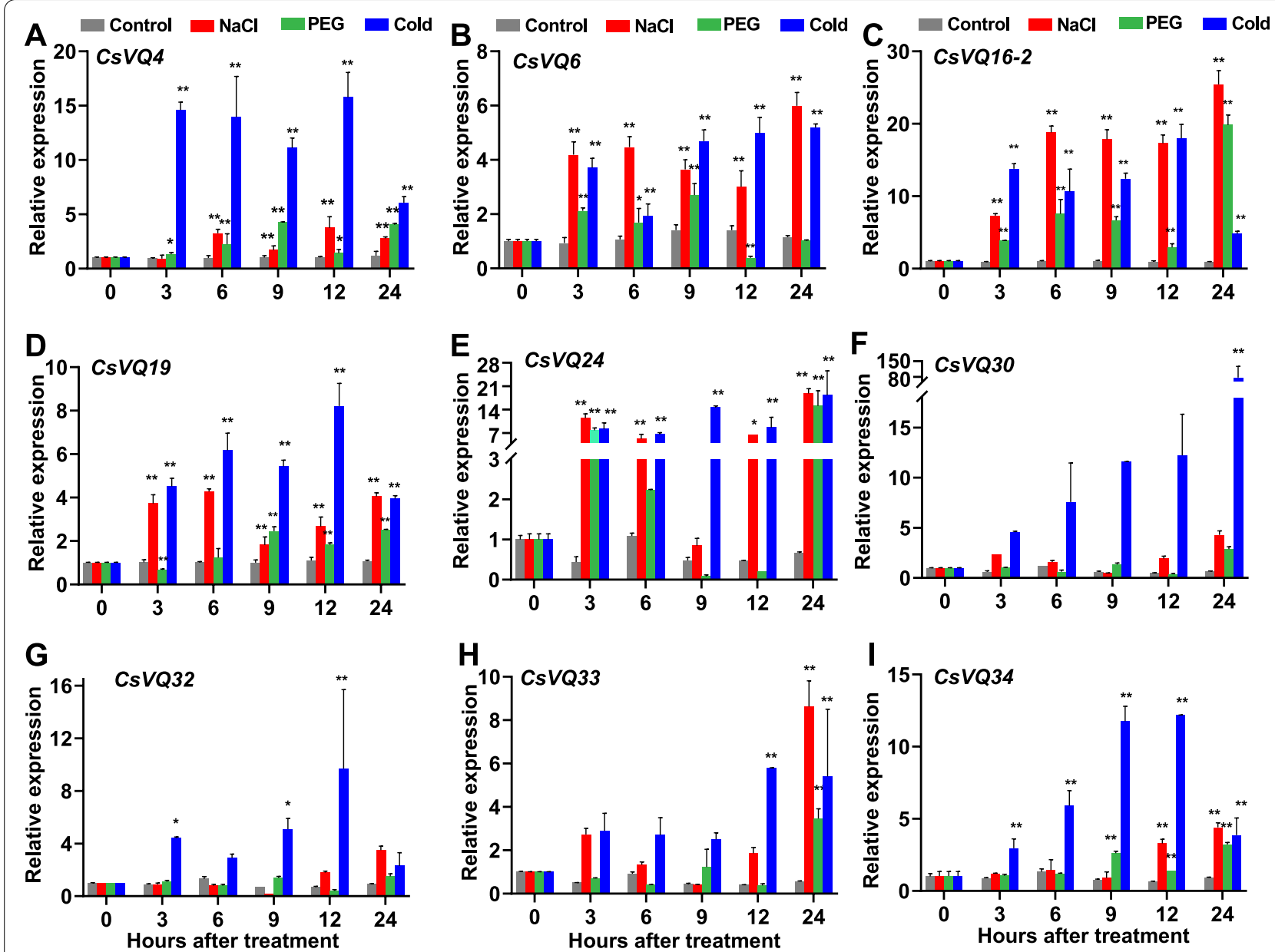

Fig. 8 Gene expression analysis of certain CsVQ genes under abiotic stress. Expression of CsVQ4 (A), CsVQ6 (B), CsVQ16-2 (C), CsVQ19 (D), CsVQ24 (E), CsVQ30 (F), CsVQ32 (G), CsVQ33 (H), and CsVQ34 (I) under salt (red column), drought (green column), and cold (blue column) treatments were analyzed respectively. Bars represent means \pm standard deviations $(n=3)$; data were analyzed by one-way ANOVA followed by Tukey's test (** $P<0.01$, $\left.{ }^{*} P<0.05\right)$. Accession numbers of the gene sequences used for expression analysis are listed in Table 1 , and the primers used for qRT-PCR were shown in Supplementary Table S2

inferred evolutionary relationships of these CsVQ proteins. The evolution of the $V Q$ gene family is relatively conservative, and a majority of $V Q$ genes lose introns during evolution [10], such as in Arabidopsis [6], Chinese cabbage [9], tomato [11], melon [16], and rice [7]. Similar to these findings, most CsVQ genes (81.25\%) possessed one exon structure, except that CsVQ2, CsVQ4, CsVQ7, CsVQ9, CsVQ25, and CsVQ27 contained two exon structures (Fig. S1). In addition, most of $V Q$ genes encode relatively small proteins with amino acids less than 300 amino acids, with the exception of OsVQ34 (1138 aa in Rice), $M d V Q 13$, and MdVQ48 (1076 aa, 2064 aa in Moso bamboo) [10, 17]. In cucumber, almost all CsVQ proteins contain less than 400 amino acids (Table 1). Furthermore, most of VQ proteins are predicted to be located in the nucleus and a few are positioned on the cytoplasm, chloroplast and mitochondria [6, 13, 17]. These researches indicate that VQ proteins in different plants own similarities.

$V Q$ genes have been demonstrated to participate in various aspects of growth, development and response to abiotic stresses [4]. Cis-acting stress-responsive elements in the promoter region, such as ABRE, W-box, and TCA-element, can well reflect these aspects [46]. Previous and our present studies showed that cis-elements exist in response to stress, hormones, and light, indicating the regulation of $V Q$ genes to plant growth, stress, hormones, or light $[10,15,16]$. In addition, there is growing evidence that plant VQ proteins achieve biological functions by interacting with proteins such as WRKY transcription factors, one of the largest transcriptional regulatory families that regulate plant growth and 
development [6, 15, 19, 21, 30, 34, 35, 47]. In Arabidopsis, AtVQ16, AtVQ23 act as activator of AtWRKY33 to positively regulate the plant defense [34], and AtVQ9 forms a complex with AtWRKY8 to mediate the salt response [25]. In banana, MaVQ5 might act as a repressor of MaWRKY26 in the regulation of JA biosynthesis in response to cold stress [30]. In bamboo, PeVQ28 and WRKY83 interacted in the nucleus, and the overexpression of PeVQ28 in Arabidopsis increased resistance to salt stress and enhanced sensitivity to ABA [31]. In the present analysis, W-box elements, the binding sites of WRKY proteins, were identified in the promoters of 16 CsVQ genes, with an average frequency of 0.7 (Table. S1). The value was similar to that of $C m V Q$, and lower than that of the AtVQ and $V v V Q[6,15]$. In Arabidopsis, apple, and soybean, VQ proteins only physically interact with WRKY transcription factors of Group I or Group IIc [6, $8,14]$. Twelve CsWRKYs of group I and group IIc, which were highly conserved with the corresponding Arabidopsis WRKY proteins, were presumed to interact with different CsVQ proteins (Fig. 5).

SA and MeJA are important endogenous signals mediating plant defenses [12, 48]. Previous studies have indicated that $V Q$ genes are regulated by SA and MeJA treatments $[6,10,17,24]$, implying that they are likely involved in the responses to defense-related hormones. $V Q$ genes in Arabidopsis [6], strawberry [12], and melon [16] were demonstrated to be responsive to SA and MeJA treatments to varying degrees. In $A$. thaliana, AtVQ21, AtVQ22 can be inhibited by SA, but activated by JA $[6,25,49]$, while $A t V Q 23$ can be induced by both SA and JA treatments [6, 34]. CmVQ21-1, CmVQ22-1, and $C m V Q 23$ in melon displayed similar trends with $A t V Q 21, A t V Q 22$, and $A t V Q 23$, respectively. In the present study, the highly expressed $C s V Q$ genes, including CsVQ4, CsVQ6, CsVQ16-2, CsVQ19, CsVQ24, and $C s V Q 34$, were induced by MeJA and SA to varying degrees (Fig. 7), indicating that they might play crucial roles in SA- and JA-regulated defense. Almost all highly expressed $C s V Q$ genes are involved in the response to hormones, indicating that $C s V Q s$ may participate in the regulation of cucumber abiotic stresses through different hormone-mediated signal transduction pathways.

Low temperature, salinity, and drought are common abiotic stresses that impair plant growth and development. Exogenous application of ABA to higher plants can accelerate their adaptation to these stresses by changing the expression of resistance genes [50], particularly for $C s V Q$ genes (CsVQ4, CsVQ6, CsVQ16-2, CsVQ19, CsVQ24, CsVQ30, CsVQ32, CsVQ33, and CsVQ34) with higher expression levels in different tissues (Fig. 6). CsVQ4 is homologous to AtVQ4 (MVQ1) and SlVQ6, both of which are involved in the response to salt and drought stresses [11,
18]. In our analysis, CsVQ4, CsVQ6, CsVQ16-2, CsVQ19, and $C s V Q 24$ were significantly upregulated by low temperature, salinity, drought, and ABA treatment (Figs. 7 and 8). Similar results were obtained for ABA-treated $V Q$ genes in rice, Moso bamboo, and melon [10, 16, 17]. CsVQ16-2 exhibited drastically similar up-expression trends under abiotic stress and ABA treatments (Fig. 7). In cucumber, CsVQ16-2 responded to salt, drought, and cold stresses (Fig. 8). In Arabidopsis, AtVQ16 (SIB2), homologous to CsVQ16-2 in cucumber, counteracts adversity by specifically identifying the C-terminal WRKY domain, which acts as a co-activator of AtWRKY33 in plant defense [34]. These results suggest that these $C s V Q$ genes might participate in the ABA-dependent signaling transaction pathway to enhance plant adaptation to abiotic stresses. Further studies are required to identify presumed VQ interacting proteins and elucidate the signaling pathways in which they are involved.

\section{Conclusions}

In conclusion, this study provides the comprehensive and systematic analysis of the $V Q$ gene family in cucumber plants. A total of $32 \mathrm{VQ}$ motif-containing proteins were identified and divided into 9 groups in cucumber. Genomewide bioinformatics of $C s V Q$ genes were performed to study the gene characteristics, evolution, and interaction networks. Furthermore, expression profiles of $C s V Q$ genes were carried out to determine their potential functions in the growth, development and stress response in cucumber plant. $C s V Q$ genes play an active part in the regulation of growth development, and response to salt, drought, and cold stress of cucumber plant, which may be closely related to the interactions of CsVQ proteins with CsWRKY transcription factors. These results will provide a basis for further research on biological functional differentiation, molecular mechanisms, and the role of $V Q$ genes in abiotic stress response in cucumber.

\section{Methods}

\section{Plant materials and stress treatments}

Cucumber (C. sativus cv. Xintaimici, donated by the Prof. Xiaolei Sui from China Agricultural University) plants were grown in a phytotron for $16 / 8 \mathrm{~h}$ with day/night temperatures set at $25 / 18^{\circ} \mathrm{C}$. Cucumber plants at the 3-4 true-leaf stage were used for the stress and hormone treatments. For salt and drought stress, $250 \mathrm{mM}$ sodium chloride and $20 \%(\mathrm{w} / \mathrm{v})$ PEG 6000, instead of water, were used to irrigate cucumber seedlings respectively. For low-temperature treatment, seedlings were exposed to $6^{\circ} \mathrm{C}$ for $48 \mathrm{~h}$. For hormone treatment, leaves were sprayed with $100 \mu \mathrm{M}$ ABA, $200 \mu \mathrm{M} \mathrm{SA}$, and $175 \mu \mathrm{M}$ MeJA [51]. The first and second true leaves of treated cucumber plants were randomly collected at $0,1,3,6,9,12$, and $24 \mathrm{~h}$ after treatment, then 
rapidly frozen in liquid nitrogen, and stored at $-80^{\circ} \mathrm{C}$ until analysis.

\section{Sequence retrieval and identification, chromosomal location, gene structure, and promoter analysis of CsVQ genes}

To identify the $V Q$ genes in cucumber plants, VQ proteins from A. thaliana [6] were used as queries in cucumber 'Chinese Long' v2 genome (http://www.cucurbitge nomics.org/organism/2) in the CuGenDB (http://www. cucurbitgenomics.org/) [37, 52]. In addition, motif ID "PF05678" was used as the keyword for query in the cucumber database, and VQ motif-containing proteins were rechecked and confirmed by using the InterPro program (http://www.ebi.ac.uk/inter pro/) and SMART program (http://smart.embl.de/).

The length, molecular weight (MW), and theoretical isoelectric point ( $\mathrm{pI}$ ) of VQ proteins were calculated using the ProtParam tool (https://web.expasy.org/protp aram/). The chromosomal locations of the CsVQ genes were determined using MapChart 2.3.2 [53]. Intron/exon structure analysis was performed using the Gene Structure Display Server (http://gsds.cbi.pku.edu.cn). CDS and genomic sequences of $C s V Q$ genes were submitted to obtain the gene structure and draw diagram. Subcellular locations of $\mathrm{CsVQ}$ proteins were predicted using the EukmPLoc 2.0 server (http://www.csbio.sjtu.edu.cn/bioinf/ euk-multi-2/) [54].

A sequence of $1500 \mathrm{bp}$ upstream from the start codon of each CsVQ gene was downloaded from cucumber genome. Then cis-elements in promoter of each CsVQ gene were predicted by using the PlantCARE server (http://bioinformatics.psb.ugent.be/webtools/plantcare/ $\mathrm{html} /$ ) [55].

\section{Sequence alignment, conserved motif, and phylogenetic analysis}

Amino acid sequences of the VQ proteins in cucumber were aligned by using DNAMAN version 9.0 (LynnonBiosoft, Quebec, Canada) [56]. Thirty-four Arabidopsis $\mathrm{VQ}$ protein sequences and 40 rice $\mathrm{VQ}$ protein sequences were downloaded from the TAIR database (https://www. arabidopsis.org/) and rice data sites (http://www.ricedata. $\mathrm{cn} /$ gene/), respectively. By using the maximum likelihood method [57] in MEGA X [58], the full-length sequences of VQ proteins from cucumber, Arabidopsis, rice, and tomato were compared to construct a phylogenetic tree. The distribution of conserved motifs of CsVQ in cucumber was analyzed using the MEME website (http://memesuite.org/) with 10 maximum numbers of motifs.

\section{Analysis of interaction networks of CsVQ proteins}

Functional interaction network models of CsVQ proteins were established using the STRING database (https:// string-db.org), and the confidence parameters were set as 0.40 threshold. The interaction between CsVQs and CsWRKYs was predicted using PAIR website (http:// www.cls.zju.edu.cn/pair/), and the interaction network was constructed using Cytoscape 3.7.2 [59].

\section{RNA extraction and CDNA reverse transcription}

Total RNA was isolated from the leaves of cucumber plants using an RNA Extraction Kit (Huayueyang, China) according to the manufacturer's instructions. Briefly, 50 100 mg of leaf tissues were quickly ground into powder in liquid nitrogen, transferred into Eppendorf tubes, and then immediately mixed by vortexing in lysis buffer. Prior to centrifuging at $12,000 \mathrm{rpm}$ for $10 \mathrm{~min}$ at $4{ }^{\circ} \mathrm{C}$, chloroform was added, blended by vortexing for $15 \mathrm{~s}$, and incubated for $3 \mathrm{~min}$ at room temperature to phase separation. After transferring supernatant to new RNase-free tubes, potential RNA was precipitated, thoroughly rinsed by wash buffer, and then eluted by adding RNase-free water onto the membrane of spin column. Concentration and purity of total RNA were assessed in a NanoDrop 2000c Spectrophotometer (Thermo Scientific, USA), using a $1 \mu \mathrm{l}$ aliquot of the total RNA solutions. RNA purity was estimated from the A260 $\backslash$ A280 absorbance ratio. The integrity of total RNA was evaluated by running samples on $1.5 \%$ agarose gels. The ratio of the peak areas (28 $\quad \mathrm{S} \backslash 18 \mathrm{~S})$ corresponding to the $28 \mathrm{~S}$ and $18 \mathrm{~S}$ ribosomal RNAs was used as a reference for RNA degradation.

Reverse transcription was performed using a Hifair ${ }^{\circledR}$ II 1st Strand cDNA Synthesis Kit (gDNA digester plus, Yeasen, China) according to the manufacturer's protocol. Total RNA samples were treated with RNase-free DNase and used for cDNA synthesis. The transcripts of $C s V Q$ genes were analyzed by qRT-PCR using $\mathrm{SYBR}^{\circledR}$ Premix Ex $\operatorname{Taq}^{\mathrm{TM}}$ II (TakaRa, Japan) on a Bio-Rad IQ5 Real-Time PCR System (Bio-Rad, USA). Gene-specific primers (Supplement Table S2) were designed by using Primer Premier 6. The PCR conditions consisted of denaturation at $95^{\circ} \mathrm{C}$ for $30 \mathrm{~s}$, followed by 40 cycles of $95^{\circ} \mathrm{C}$ for $5 \mathrm{~s}$, and $60^{\circ} \mathrm{C}$ for $34 \mathrm{~s}$. For relative quantification, the cucumber $\alpha$-TUBULIN gene was used as the internal control, and the relative expression levels were repeated in triplicate using the $2^{-\Delta \Delta C T}$ method [60].

\footnotetext{
Abbreviations

ABA: Abscisic acid; CuGenDB: Cucurbit Genomics Database; JA: Jasmonic acid; MeJA: Methyl jasmonate; qRT-PCR: Quantitative real-time polymerase chain reaction; SA: Salicylic acid; VQ: Valine-glutamine.
} 


\section{Supplementary Information}

The online version contains supplementary material available at https://doi. org/10.1186/s12870-021-03242-9.

Additional file 1: Figure S1. Intron and exon structures of the VQ genes in cucumber. The majority of the CSVQ genes only have one exon, except CsVQ2, CsVQ4, CsVQ7, CsVQ9, CsVQ25, and CsVQ27, which have two exons, Figure S2. Cis-elements in the promoters of CSVQ genes. Supplementary Table S1. Number of cis-elements related to various environmental stresses in the promoters of CSVQ genes. Supplementary Table S2. Primers used for quantitative PCR analysis in this study.

\section{Acknowledgments}

The authors thank all contributors for their work and would like to thank the reviewers for their valuable comments and suggestions.

\section{Authors' contributions}

Z.G., Q.Z., and N.S. designed the project. N.S., Z.X., J.S., Y.X., P.W., and X.C. performed the experiments. Q.Z. performed bioinformatics analysis. N.S. and Z.G. wrote the manuscript. Z.X., J.S., Y.X., P.W., and X.C. provided useful advice. Al authors read and approved the final manuscript.

\section{Funding}

This work was supported by National Natural Science Foundation of China (31960591), Youth Science Foundation of Jiangxi Science and Technology Department (20202BABL215010), and Science and Technology Foundation of Jiangxi Provincial Department of Education (GJJ180219). The funding agent only provided the financial support and did not involve in the design of the experiment, collection, interpretation and analysis of data and in the drafting of the manuscript.

\section{Availability of data and materials}

Most data generated or analysed during this study are included in this article and its supplemental files. The sequencing data (bioproject PRJNA312872 of Cucurbit Expression Atlas) used and analyzed during this study is available in the Cucurbit Genomics Database.

\section{Declarations}

Ethics approval and consent to participate

Not applicable.

\section{Consent for publication}

Not applicable.

\section{Competing interests}

The authors declare no competing interests.

Received: 28 June 2021 Accepted: 20 September 2021

Published online: 25 October 2021

\section{References}

1. Arab MM, Marrano A, Abdollahi-Arpanahi R, Leslie CA, Cheng H, Neale $\mathrm{DB}$, et al. Combining phenotype, genotype, and environment to uncover genetic components underlying water use efficiency in Persian walnut. J Exp Bot. 2019;71(3):1107-27.

2. Jing $Y$, Lin R. The VQ motif-containing protein family of plant-specific transcriptional regulators. Plant Physiol. 2015;169(1):371-8.

3. Arab MM, Marrano A. Genome-wide patterns of population structure and association mapping of nut-related traits in Persian walnut populations from Iran using the Axiom J. regia 700K SNP array. Sci Rep-UK. 2019;9(1):6376.

4. Yuan G, Qian Y, Ren Y, Guan Y, Wu X, Ge C, et al. The role of plant-specific VQ motif-containing proteins: an ever-thickening plot. Plant Physiol Biochem. 2021;159:12-6.
5. Morikawa K, Shiina T, Murakami S, Toyoshima Y. Novel nuclear-encoded proteins interacting with a plastid sigma factor, Sig1, in Arabidopsis thaliana1 1 Nucleotide sequence data reported are available in the GenBank databases under the accession number AF224762. FEBS Lett. 2002;514(2):300-4.

6. Cheng Y, Zhou Y, Yang Y, Chi YJ, Zhou J, Chen JY, et al. Structural and functional analysis of $V Q$ motif-containing proteins in Arabidopsis as interacting proteins of WRKY transcription factors. Plant Physiol. 2012;159(2):810-25.

7. Li N, Li X, Xiao J, Wang S. Comprehensive analysis of VQ motif-containing gene expression in rice defense responses to three pathogens. Plant Cell Rep. 2014;33(9):1493-505.

8. Zhou Y, Yang Y, Zhou X, Chi Y, Fan B, Chen Z. Structural and functional characterization of the VQ protein family and VQ protein variants from soybean. Sci Rep-UK. 2016;6(1):34663.

9. Zhang G, Wang F, Li J, Ding Q, Zhang Y, Li H, et al. Genome-wide identification and analysis of the VQ motif-containing protein family in Chinese cabbage (Brassica rapa L. ssp. Pekinensis). Int J Mol Sci. 2015;16(12):28683-704.

10. Wang Y, Liu H, Zhu D, Gao Y, Yan H, Xiang Y. Genome-wide analysis of VQ motif-containing proteins in Moso bamboo (Phyllostachys edulis). Planta. 2017;246(1):165-81.

11. Ding H, Yuan G, Mo S, Qian Y, Ge C. Genome-wide analysis of the plantspecific VQ motif-containing proteins in tomato (Solanum lycopersicum) and characterization of SIVQ6 in thermotolerance. Plant Physiol Biochem. 2019;143.

12. Garrido-Gala J, Higuera JJ, Muñoz-Blanco J, Amil-Ruiz F, Caballero JL. The VQ motif-containing proteins in the diploid and octoploid strawberry. Sci Rep-UK. 2019;9(1):4942.

13. Guo J, Chen J, Yang J, Yu Y, Yang Y, Wang W. Identification, characterization and expression analysis of the VQ motif-containing gene family in tea plant (Camellia sinensis). BMC Genomics. 2018;19(1):710.

14. Dong Q, Zhao S, Duan D, Tian Y, Wang Y, Mao K, et al. Structural and functional analyses of genes encoding VQ proteins in apple. Plant Sci. 2018:272:208-19.

15. Wang M, Vannozzi A, Wang G, Zhong Y, Corso M, Cavallini E, Cheng $Z-M$. A comprehensive survey of the grapevine VQ gene family and its transcriptional correlation with WRKY proteins. Front Plant Sci 2015;6:417

16. Zhang G, Wei B. Characterization of VQ motif-containing protein family and their expression patterns under phytohormones and abiotic stresses in melon (Cucumis melo L.). Plant Growth Regul. 2019;89(3):273-85.

17. Kim DY, Kwon Sl, Choi C, Lee H, Ahn I, Park SR, et al. Expression analysis of rice VQ genes in response to biotic and abiotic stresses. Gene. 2013;529(2):208-14

18. Pecher P, Eschen-Lippold L, Herklotz S, Kuhle K, Naumann K, Bethke G, et al. The Arabidopsis thaliana mitogen-activated protein kinases MPK3 and MPK6 target a subclass of 'VQ-motif'-containing proteins to regulate immune responses. New Phytol. 2014;203(2):592-606.

19. Lei R, Li X, Ma Z, Lv Y, Hu Y, Yu D. Arabidopsis WRKY2 and WRKY34 transcription factors interact with VQ20 protein to modulate pollen development and function. Plant J. 2017;91(6):962-76.

20. Wang A, Garcia D, Zhang H, Feng K, Chaudhury A, Berger F, et al. The VQ motif protein IKU1 regulates endosperm growth and seed size in Arabidopsis. Plant J. 2010;63(4):670-9.

21. Uji Y, Kashihara K, Kiyama H, Mochizuki S, Akimitsu K, Gomi K. Jasmonic acid-induced VQ-motif-containing protein OsVQ13 influences the OsWRKY45 signaling pathway and grain size by associating with OsMPK6 in rice. Int J Mol Sci. 2019;20(12):2917.

22. Pan J, Wang H, Hu Y, Yu D. Arabidopsis VQ18 and VQ26 proteins interact with $\mathrm{ABI} 5$ transcription factor to negatively modulate ABA response during seed germination. Plant J. 2018;95(3):529-44.

23. Li Y, Jing Y, Li J, Xu G, Lin R. Arabidopsis VQ MOTIF-CONTAINING PROTEIN 29 represses seedling deetiolation by interacting with PHYTOCHROMEINTERACTING FACTOR1. Plant Physiol. 2014;164(4):2068-80.

24. Wang H, Hu Y, Pan J, Yu D. Arabidopsis VQ motif-containing proteins VQ12 and VQ29 negatively modulate basal defense against Botrytis cinerea. Sci Rep-UK. 2015;5(1):14185.

25. Hu P, Zhou W, Cheng Z, Fan M, Wang L, Xie D. JAV1 controls jasmonateregulated plant defense. Mol Cell. 2013;50(4):504-15. 
26. Li X, Qin R, Du Q, Cai L, Hu D, Du H, et al. Knockdown of GmVQ58 encoding a VQ motif-containing protein enhances soybean resistance to the common cutworm (Spodoptera litura Fabricius). J Exp Bot. 2020;71(10):3198-210

27. Zou Z, Liu F, Huang S, Fernando D. Genome-wide identification and analysis of VQ motif-containing gene family in Brassica napus and functional characterization of BnMKS1 in response to Leptosphaeria maculans. Phytopathology. 2020;111.

28. Hu Y, Chen L, Wang H, Zhang L, Wang F, Yu D. Arabidopsis transcription factor WRKY 8 functions antagonistically with its interacting partner VQ9 to modulate salinity stress tolerance. Plant J. 2013;74(5):730-45.

29. Perruc E, Charpenteau M, Ramirez BC, Jauneau A, Galaud J-P, Ranjeva R, et al. A novel calmodulin-binding protein functions as a negative regulator of osmotic stress tolerance in Arabidopsis thaliana seedlings. Plant J. 2004;38(3):410-20.

30. Ye Y-J, Xiao Y-Y, Han Y-C, Shan W, Fan Z-Q, Xu Q-G, et al. Banana fruit VQ motif-containing protein 5 represses cold-responsive transcription factor MaWRKY26 involved in the regulation of JA biosynthetic genes. Sci RepUK. 2016;6(1):23632

31. Cheng $X$, Wang $Y$, Xiong $R$, Gao $Y$, Yan $H$, Xiang Y. A Moso bamboo gene VQ28 confers salt tolerance to transgenic Arabidopsis plants. Planta. 2020:251(5):99.

32. Zhu H, Zhou Y, Zhai H, He S, Zhao N, Liu Q. A novel Sweetpotato WRKY transcription factor, IbWRKY2, Positively Regulates Drought and Salt Tolerance in Transgenic Arabidopsis. Biomolecules. 2020;10:506.

33. Song W, Zhao H, Zhang X, Lei L, Lai J. Genome-wide identification of VQ motif-containing proteins and their expression profiles under abiotic stresses in maize. Front Plant Sci. 2016;6(281):1177.

34. Lai Z, Li Y, Wang F, Cheng Y, Fan B, Yu J-Q, et al. Arabidopsis sigma factor binding proteins are activators of the WRKY33 transcription factor in plant defense. Plant Cell. 2011:23(10):3824-41.

35. Chen J, Wang H, LiY, Pan J, Hu Y, Yu D. Arabidopsis VQ10 interacts with WRKY8 to modulate basal defense against Botrytis cinerea. J Integr Plant Biol. 2018;60(10):956-69.

36. Ali MRM, Uemura T, Ramadan A, Adachi K, Nemoto K, Nozawa A, et al. The ring-type E3 ubiquitin ligase JUL1 targets the VQ-motif protein JAV1 to coordinate jasmonate signaling. Plant Physiol. 2018;179(4):1273-84.

37. Huang S, Li R, Zhang Z, Li L, Gu X, Fan W, et al. The genome of the cucumber, Cucumis sativus L. Nat Genet. 2009;41(12):1275-81.

38. Li Z, Zhang Z, Yan P, Huang S, Fei Z, Lin K. RNA-Seq improves annotation of protein-coding genes in the cucumber genome. BMC Genomics. 2011;12(1):540.

39. Sadat-Hosseini M, Bakhtiarizadeh MR, Boroomand N, Tohidfar M, Vahdat K. Combining independent de novo assemblies to optimize leaf transcriptome of Persian walnut. PLoS One. 2020;15:e0232005.

40. Li Q, Li H, Huang W, Xu Y, Zhou Q, Wang S, et al. A chromosome-scale genome assembly of cucumber (Cucumis sativus L.). GigaScience. 2019;8(6):1-10.

41. Ling J, Jiang W, Zhang Y, Yu H, Mao Z, Gu X, et al. Genome-wide analysis of WRKY gene family in Cucumis sativus. BMC Genomics. 2011;12(1):471.

42. Zhang $L$, Lv D, Pan J, Zhang $K$, Wen $H$, Chen $Y$, et al. A SNP of HD-ZIP I transcription factor leads to distortion of trichome morphology in cucumber (Cucumis sativus L.). BMC Plant Biol. 2021:21(1):182.

43. Wang $X$, Zhang $H$, Sun G, Jin Y, Qiu L. Identification of active VQ motifcontaining genes and the expression patterns under low nitrogen treatment in soybean. Gene. 2014;543(2):237-43.

44. Zhong Y, Guo C, Chu J, Liu H, Cheng Z-M, Crease T. Microevolution of the VQ gene family in six species of Fragaria. Genome. 2018;61(1):49-57.
45. Azzarito V, Long K, Murphy NS, Wilson AJ. Inhibition of a-helix-mediated protein-protein interactions using designed molecules. Nat Chem. 2013:5(3):161-73.

46. Zhao J, Gao Y, Zhang Z, Chen T, Guo W, Zhang T. A receptor-like kinase gene (GbRLK) from Gossypium barbadense enhances salinity and drought-stress tolerance in Arabidopsis. BMC Plant Biol. 2013;13(1):110.

47. Weyhe M, Eschen-Lippold L, Pecher P, Scheel D, Lee J. Ménage à trois: the complex relationships between mitogen-activated protein kinases, WRKY transcription factors, and VQ-motif-containing proteins. Plant Signal Behav. 2014;9(8):e29519.

48. Shilina JV, Gushcha MI, Molozhava OS, Litvinov SV, Dmitriev AP. Induction of Arabidopsis thaliana resistance to pathogenic bacteria by lipopolysaccharide and salicylic acid. Cytol Genet. 2018;52(3):169-73.

49. Fiil BK, Petersen M. Constitutive expression of MKS1 confers susceptibility to Botrytis cinerea infection independent of PAD3 expression. Plant Signal Behav. 2011;6(10):1425-7.

50. Sah SK, Reddy KR, Li J. Abscisic acid and abiotic stress tolerance in crop plants. Front Plant Sci. 2016;7:571.

51. Wan H, Zhao Z, Malik AA, Qian C, Chen J. Identification and characterization of potential NBS-encoding resistance genes and induction kinetics of a putative candidate gene associated with downy mildew resistance in Cucumis. BMC Plant Biol. 2010;10(1):186.

52. Cucurbit Genomics Database http://www.cucurbitgenomics.org/. Accessed 20 May 2021

53. Voorrips RE. MapChart: software for the graphical presentation of linkage maps and QTLs. J Hered. 2002;93(1):77-8.

54. Chou KC, Shen HB. A new method for predicting the subcellular localization of eukaryotic proteins with both single and multiple sites: Euk-mPLoc 2.0. PLoS One. 2010;5(4):e9931.

55. Lescot M, Déhais P, Thijs G, Marchal K, Moreau Y, Van de Peer $Y$, et al. PlantCARE, a database of plant cis-acting regulatory elements and a portal to tools for in silico analysis of promoter sequences. Nucleic Acids Res. 2002;30(1):325-7.

56. Wilbur W, Lipman D. Rapid similarity searches of nucleic acid and protein data banks. Proc Natl Acad Sci U S A. 1983;80:726-30.

57. Kishino $\mathrm{H}$, Hasegawa M. Evaluation of the maximum likelihood estimate of the evolutionary tree topologies from DNA sequence data, and the branching order in hominoidea. J Mol Evol. 1989:29(2):170-9.

58. Kumar S, Stecher G, Li M, Knyaz C, Tamura K. MEGA X: molecular evolutionary genetics analysis across computing platforms. Mol Biol Evol. 2018;35(6):1547-9.

59. Shannon P, Markeil A, Ozier O, Baliga NS, Wang JT, Ramage D, et al. Cytoscape: a software environment for integrated models of biomolecular interaction networks. Genome Res. 2003;13(11):2498-504.

60. Livak KJ, Schmittgen TD. Analysis of relative gene expression data using real-time quantitative PCR and the $2^{-\triangle \Delta C T}$ method. Methods. 2001:25(4):402-8

\section{Publisher's Note}

Springer Nature remains neutral with regard to jurisdictional claims in published maps and institutional affiliations.

Ready to submit your research? Choose BMC and benefit from:

- fast, convenient online submission

- thorough peer review by experienced researchers in your field

- rapid publication on acceptance

- support for research data, including large and complex data types

- gold Open Access which fosters wider collaboration and increased citations

- maximum visibility for your research: over 100M website views per year

At BMC, research is always in progress.

Learn more biomedcentral.com/submissions 